\title{
On the relationship between categorical frequency estimation and cued recall
}

\author{
ROBERT L. GREENE \\ Case Western Reserve University, Cleveland, Ohio
}

\begin{abstract}
In a categorical frequency-estimation task, subjects have to indicate the number of exemplars from particular categories that have been shown on a list. In three experiments, the relationship between this task and the recall of exemplars with category names as cues was investigated. Three variables (generation, blocking of category exemplars, and provision of extralist cues) were shown to have identical effects on the two tasks. These results support a model of categorical frequency estimation in which subjects use a category name to retrieve exemplars and then base their frequency estimate on a count of the exemplars that have been retrieved.
\end{abstract}

In experiments testing categorical frequency estimation, subjects are shown a list composed of exemplars from several taxonomic categories. Typically, each exemplar is presented once. At the time of the test, the subjects are shown category names and asked to indicate how many exemplars of each category have been shown. Subjects do this task with considerable accuracy (e.g., Alba, Chromiak, Hasher, \& Attig, 1980; Barsalou \& Ross, 1986; Brooks, 1985; Hanson \& Hirst, 1988; Williams \& Durso, 1986). The experiments reported here test two accounts of how people do such a task.

\section{Category-Counter Account}

Alba et al. (1980) and Brooks (1985) have proposed that categorical frequency estimation is based solely upon information stored at the superordinate level. This account assumes that, when an exemplar is presented on a list, a representation of its categorical superordinate is activated. This activation leads to the incrementing of a frequency counter associated with that subordinate. When subjects are later asked to estimate how many exemplars have been presented from a particular category, they base their response on the value of the category frequency counter. Traces of individual exemplars are not retrieved. Alba et al. and Brooks also assume that the incrementing of the category counter is an automatic process unaffected by intentionality or strategy.

\section{Exemplar-Retrieval Account}

Williams and Durso (1986) have presented a very different account. They have suggested that each presentation of an exemplar forms an independent trace in memory. When subjects are given a categorical frequency test, they use each category name as a cue to retrieve the traces of the exemplars that were presented. Then, the subjects

This research was supported by a research initiation grant from Case Western Reserve University. Correspondence concerning this article should be addressed to Robert L. Greene, Department of Psychology, Case Western Reserve University, Cleveland, $\mathrm{OH} 44106$. count the number of retrieved traces and base their response on this count. Information stored at the superordinate level is not consulted. This account is related to the multiple-trace theory of item repetition effects (Hintzman, 1976), the availability heuristic of probabilistic reasoning (Tversky \& Kahneman, 1973), and exemplar theories of category representation (e.g., Hintzman, 1986; Medin \& Schaffer, 1978).

The exemplar-retrieval account sees categorical frequency estimation as being akin to cued recall (using category names as retrieval cues). The only difference occurs at the output stage. In cued recall, subjects output the exemplars that have been retrieved. In categorical frequency estimation, subjects count the retrieved exemplars and give a number as a response. This number need not be identical to the number of exemplars retrieved. Since subjects may know the range of frequencies on the list, subjects may transform these counts to have their responses cover the complete range. They may also inflate their responses to adjust for exemplars that they suspect they have failed to retrieve. However, there should be a monotonic relationship between the number of retrieved traces and output response. Therefore, any variable that facilitates cued recall should lead to higher categorical frequency estimates.

\section{Preview of the Experiments}

In this study, three variables (item generation, exemplar spacing, and extralist cuing) are manipulated. Since all three variables have been shown to influence cued recall, the exemplar-retrieval account makes the prediction that categorical frequency estimation should also be affected by them. In contrast, as will be explained below, the category-counter approach predicts that none of these variables should influence categorical frequency estimation.

\section{EXPERIMENT 1}

There is an advantage of memory for material that is self-generated (Slamecka \& Graf, 1978). This generation 
effect is found in many memory tasks, including cued recall (e.g., Begg \& Snider, 1987; Slamecka \& Graf, 1978). Increasingly, generation is being seen as having its effects by inducing subjects to devote greater rehearsal (Slamecka \& Katsaiti, 1987) or mental processing (Begg \& Snider, 1987; McFarland, Frey, \& Rhodes, 1980) to generated material than to copied material.

The exemplar-retrieval account predicts that the effects of generation in categorical frequency estimation should resemble those found in cued recall: frequency estimates should be higher for categories with generated exemplars than for categories with copied exemplars. In contrast, to the extent that the effects of generation are mediated by rehearsal or mental effort, the category-counter approach would predict that categorical frequency estimation should be unaffected by generation. This prediction stems from Alba et al.'s (1980) and Brooks's (1985) assumption that the incrementing of a category frequency counter occurs automatically and should therefore be uninfluenced by rehearsal or effort.

\section{Method}

Subjects. The participants were 48 introductory psychology students at Case Western Reserve University fulfilling a course requirement. All were tested individually.

Materials. The stimuli came from 32 taxonomic categories, selected from the Battig and Montague (1969) and Hunt and Hodge (1971) norms. The first four exemplars from each category were used, except in cases of ambiguity (e.g., ORANGE could be either a fruit or a color), in which case a lower exemplar was selected.

Eight lists were constructed. Each list consisted of 1 to 4 exemplars from each of the 32 categories. Exemplars from a single category were separated by 5 to 10 exemplars from other categories. Five primacy and 5 recency buffers from other categories were added, so that each list consisted of 90 items.

All list items were printed on sheets of paper. The items from half of the categories were printed in uppercase letters. These items were used in the copy condition. The first two letters of each item from the other categories were printed in lowercase and in reverse order. These items were used in the generation condition. Across the eight lists, all the categories were used at each frequency in both the copy and the generation conditions.

Procedure. At the beginning of the experiment, the subjects were told that they would be shown a list of words and given an unspecified memory test. Then they received 90 blank index cards. The list items were presented individually on sheets of paper at a 5-sec rate. If a word was printed normally, the subjects had to copy it on a card and then turn the card face down. If a word had its first two letters backwards in lowercase, the subjects had to reverse the first two letters mentally and then write the word. This task has been used in other generation studies (Greene, 1988; Nairne, Pusen, \& Widner, 1985; Nairne \& Widner, 1987).

After the list had been shown, the subjects were given a sheet of paper with the names of the 32 taxonomic categories. Half of the subjects were given cued-recall instructions; they were told to write down the items from each of these categories that had been shown earlier. The other subjects were given categorical frequencyestimation instructions; they were told to print a number from one to four to indicate how many exemplars had been shown from that category

\section{Results and Discussion}

In all significance tests, a significance level of .05 was used unless otherwise noted. The number of items recalled
Table 1

Mean Performance per Category in Experiment 1

\begin{tabular}{lcccc}
\hline & \multicolumn{4}{c}{ Category Frequency } \\
\cline { 2 - 4 } \multicolumn{1}{c}{ Test } & 1 & 2 & 3 & 4 \\
\hline Cued recall & & & & \\
$\quad$ Copied & 0.62 & 0.95 & 1.75 & 2.05 \\
$\quad$ Generated & 0.67 & 1.25 & 2.14 & 2.68 \\
Frequency estimates & & & & \\
$\quad$ Copied & 1.25 & 1.63 & 2.29 & 2.68 \\
$\quad$ Generated & 1.30 & 1.80 & 2.51 & 3.21 \\
\hline
\end{tabular}

per category and the magnitude of frequency estimates per category are shown in Table 1. The recall data, however, should be interpreted with caution. The number of items recalled per category increases with category frequency, because there were more items to recall at higher frequencies. The recall data are presented in terms of number recalled rather than proportions, in order to facilitate comparison with the frequency estimates.

The exemplar-retrieval account predicts that the results from the frequency task should parallel those found in recall. The data support this prediction, with frequency and generation having equivalent effects on the two tasks. An analysis of variance was made, with type of test (recall or frequency), category frequency, and condition (copy or generation) as independent variables. The effect of test was significant $\left[F(1,46)=26.66, M S_{\mathrm{e}}=18.83\right]$, reflecting the fact that estimates were higher than numbers of items recalled. This is not surprising, since the subjects knew that the range of responses was from one to four on the frequency task, and they could distribute their responses accordingly even if they could not remember all of the exemplars. There were significant effects of frequency $\left[F(3,138)=234.87, M S_{\mathrm{e}}=3.76\right]$ and generation $\left[F(1,46)=30.85, M S_{\mathrm{e}}=3.90\right]$. There was a significant interaction between generation and frequency $\left[F(3,138)=6.76, M S_{\mathrm{e}}=3.16\right]$. This reflected the fact that generation had little effect at the lowest frequency (see Greene, 1988, for a similar finding with respect to item frequency estimation). Type of test did not interact with any other variable. The interactions between test and generation $\left[F(1,46)=1.60, M S_{\mathrm{e}}=3.90\right]$, between test and frequency $\left[F(3,138)=0.63, M S_{\mathrm{e}}=0.63\right]$, and among test, generation, and frequency $[F(3,138)=0.03$, $\left.M S_{\mathrm{e}}=3.16\right]$ were nonsignificant. These null interactions imply that generation and frequency affected the two tests similarly.

Analyses were also conducted separately on the two measures. The analysis of the cued-recall data exhibited significant effects of frequency $\left[F(3,69)=199.51, M S_{\mathrm{e}}=\right.$ $2.35]$ and generation $\left[F(1,23)=21.79, M S_{\mathrm{e}}=2.61\right]$, as well as a significant interaction between generation and frequency $\left[F(3,69)=4.75, M S_{\mathrm{e}}=2.19\right]$. The analysis of the magnitude of frequency estimates exhibited significant effects of frequency $\left[F(3,69)=80.51, M S_{\mathrm{e}}=5.16\right]$ and generation $\left[F(1,23)=6.91, M S_{\mathrm{e}}=5.20\right]$, and a significant interaction between frequency and generation $\left[F(3,69)=2.98, M S_{\mathrm{e}}=3.94\right]$. These analyses support the conclusion that cued recall and categorical frequency 
estimation are affected similarly by frequency and generation.

The above analyses on the frequency task were performed on the magnitude of the estimates. The exemplarretrieval account makes its most direct predictions regarding the magnitude of estimates. However, this account also suggests that the accuracy of estimates should be affected by variables that influence cued recall. The more exemplars that can be retrieved, the more accurate frequency estimation should become. If all items could be retrieved, estimation would be perfect; if no items could be retrieved, performance would be at chance. The proportion of categories given exactly the correct estimate was higher for generated categories (.47) than for copied (.37) categories $\left[F(1,23)=6.91, M S_{\mathrm{e}}=2.11\right]$. Similarly, the mean unsigned deviation between true and estimated frequency was lower for generated (.73) than for copied (1.01) categories $\left[F(1,23)=20.32, M S_{c}=11.74\right]$. Generation affects the accuracy as well as the magnitude of category frequency estimates, extending earlier results in item frequency estimation (Greene, 1988). The results of Experiment 1 are consistent with the predictions of an exemplar-retrieval account, but they conflict with the category-counter approach.

\section{EXPERIMENT 2}

One variable that typically affects cued recall is the spacing between exemplars: cued recall of exemplars is higher if they are presented in massed fashion than if they are spaced apart on a list (see Puff, 1974, for a review). This is a reversal of the normal, positive spacing effect found in studies of item repetition. Massed exemplars improve recall by facilitating the use of organizational strategies (Murphy \& Puff, 1982). Since automatic processes should not be affected by such organizational strategies, and since Alba et al. (1980) and Brooks (1985) assume that the incrementing of a category frequency counter is automatic, the category-counter approach predicts that spacing should not affect categorical frequency estimation. In contrast, the exemplar-retrieval account predicts that exemplar spacing and all other variables that influence cued recall should affect categorical frequency estimation.

Two previous studies examined this question. Alba et al. (1980, Experiment 2) found that exemplar spacing did not affect the magnitude or accuracy of frequency estimates. However, when Barsalou and Ross (1986, Experiment 3) presented items blocked either by category or by property, they found that both categorical frequency estimates and cued recall were higher when items were blocked by category (the accuracy of frequency estimates was not reported). In light of this inconsistency, Experiment 2 was designed to shed additional light on this question.

\footnotetext{
Method

Subjects. The participants were 32 Case Western Reserve University introductory psychology students fulfilling a course requirement.

Materials. The items from Experiment 1 were used. All the items were printed in uppercase letters on cards. Half of the categories
}

on each list were presented in massed fashion (i.e., consecutively). The other half of the catgories had from 5 to 10 exemplars from other categories intervening between the closest members of single categories. Across subjects, categories occurred equally often in each condition at each frequency.

Procedure. The subjects were told to attend to the list and to expect an unspecified memory test. The items were shown at a 5-sec rate; no copying or generating was required. After the items had been shown, the subjects were given either a cued-recall test or a categorical frequency-estimation test.

\section{Results and Discussion}

The mean numbers of exemplars recalled and of frequency estimates are shown in Table 2 . Since spacing was the crucial variable, analyses were performed on the data from the categories with a frequency of two or greater. As in Experiment 1, type of test had a significant effect $\left[F(1,30)=63.87, M S_{\mathrm{e}}=27.81\right]$, reflecting the fact that estimates were higher than the number actually recalled. There were significant effects of frequency $[F(2,60)=$ $\left.272.54, M S_{\mathrm{e}}=4.51\right]$ and spacing $[F(1,30)=24.81$, $\left.M S_{\mathrm{e}}=3.37\right]$. There were no significant interactions. The fact that type of test did not interact with the other variables supports the exemplar-retrieval account's prediction that these two tests should be affected similary.

An analysis of cued recall found significant effects of frequency $\left[F(2,30)=191.02, M S_{\mathrm{e}}=2.74\right]$ and spacing $\left[F(1,15)=24.28, M S_{e}=5.48\right]$. The analysis of frequency estimates likewise found effects of frequency $\left[F(2,30)=113.63, M S_{\mathrm{e}}=6.28\right]$ and spacing $[F(2,30)=$ $\left.8.33, M S_{\mathrm{e}}=15.97\right]$. Neither analysis yielded a significant interaction between frequency and spacing.

Analyses were also performed on the accuracy of frequency estimates. More categories were given the correct estimate for massed (.45) than for spaced (.36) categories $\left[F(1,15)=12.42, M S_{\mathrm{e}}=2.00\right]$, and deviations between true and estimated frequency were lower for massed $(.79)$ than for spaced $(.98)$ categories $[F(1,15)=$ $\left.18.22, M S_{\mathrm{e}}=9.89\right]$.

Exemplar spacing affected cued recall and both the magnitude and accuracy of frequency estimates. These results agree with the predictions made by an exemplar-retrieval account and with the findings of Barsalou and Ross (1986), but they conflict with those of Alba et al. (1980, Experiment 2). Alba et al. did not include a cued-recall condition, and it is possible that their manipulation of exemplar spacing would not have influenced cued recall either. Their experiment differed from the present one in a number of ways (e.g., the number of categories, and the number of exemplars per category), so that additional research will be needed to determine the causes of this discrepancy.

Table 2

Mean Performance per Category in Experiment 2

\begin{tabular}{|c|c|c|c|c|c|c|c|}
\hline \multirow[b]{3}{*}{ Test } & \multicolumn{7}{|c|}{ Category Frequency } \\
\hline & \multirow[b]{2}{*}{1} & \multicolumn{3}{|c|}{ Massed } & \multicolumn{3}{|c|}{ Spaced } \\
\hline & & 2 & 3 & 4 & 2 & 3 & 4 \\
\hline Cued recall & 0.46 & 1.25 & 1.91 & 2.24 & 0.98 & 1.53 & 2.00 \\
\hline Frequency estimates & 1.10 & 1.87 & 2.65 & 3.17 & 1.72 & 2.30 & 2.77 \\
\hline
\end{tabular}


For present purposes, the most important conclusion is that the two experiments that included both categorical frequency estimation and cued recall (Experiment 2 here and Experiment 3 in Barsalou and Ross, 1986) found parallel effects of exemplar spacing on both tasks.

\section{EXPERIMENT 3}

The first two experiments reported results consistent with the exemplar-retrieval account but inconsistent with the category-counter approach of Alba et al. (1980) and Brooks (1985). However, those experiments conflict with only one assumption of the category-counter account, namely, the assumption that incrementing of the category counter occurs automatically. This assumption of automaticity is not a necessary aspect of category-counter models. One could assume that a frequency counter is not always incremented whenever an exemplar occurs, but that it is more likely to be incremented if the subject follows a particular strategy (e.g., a strategy emphasizing semantic processing). This revised approach could explain the results of Experiments 1 and 2, as well as earlier reports of instructional effects on categorical frequency estimation (Barsalou \& Ross, 1986; Hanson \& Hirst, 1988; Williams \& Durso, 1986).

The question of whether categorical frequency estimation is based on exemplar or on superordinate information was examined more directly in Experiment 3, in which the effect of extralist cues at the time of testing was studied. The provision of category exemplars that were not on the list as retrieval cues has been shown to impair cued recall of exemplars presented on a list (Mueller \& Watkins, 1977; Watkins, 1975). Therefore, the exemplarretrieval account predicts that categorical frequency estimates should be reduced in magnitude and accuracy by the supplying of extralist cues. However, the categorycounter approach would predict no effect of extralist cuing. Since extralist cues are supplied only at the time of testing, they could not influence the encoding strategies followed by subjects. More generally, negative cuing effects reflect difficulty in retrieving items, not superordinates (Nickerson, 1984). Since the category names are always supplied to the subject at the time of the test, access to the superordinate level is presumably trivial. Therefore, any theory that attributes categorical frequency estimation solely to superordinate information would predict that the presence of extralist cues should have no effect.

\footnotetext{
Method

Subjects. The participants were 48 Case Western Reserve University introductory psychology students fulfilling a course requirement.

Materials. The four lists resembled those from Experiment 2, with the exception that all the categories were spaced (5-10 items between the closest exemplars of a category). All the categories occurred equally often at each frequency across the lists.

Procedure. The lists were presented in the same fashion as in Experiment 2. At the time of the test, the subjects were handed an answer sheet that listed all of the categories used. Under 16 categories were listed four exemplars that had not occurred on the
}

Table 3

Mean Performance per Category in Experiment 3

\begin{tabular}{lcccr}
\hline & \multicolumn{4}{c}{ Category } \\
\cline { 3 - 5 } \multicolumn{1}{c}{ Test } & 1 & 2 & 3 & 4 \\
\hline Cued recall & & & & \\
$\quad$ With extralist cues & 0.50 & 1.01 & 1.36 & 1.85 \\
$\quad$ Without extralist cues & 0.60 & 1.09 & 1.66 & 2.19 \\
Frequency estimates & & & & \\
$\quad$ With extralist cues & 1.26 & 1.46 & 2.05 & 2.94 \\
$\quad$ Without extralist cues & 1.32 & 1.72 & 2.40 & 3.17 \\
\hline
\end{tabular}

list. The other 16 categories were uncued. The subjects were asked for either cued recall or categorical frequency estimation and were told that they could use the extralist exemplars as cues. Across subjects, the categories occurred equally often in the cued and uncued conditions at each frequency.

\section{Results and Discussion}

The results are shown in Table 3. Extralist exemplars reduced both the number of exemplars recalled and the magnitude of frequency estimates. There were significant effects of test $\left[F(1,46)=38.65, M S_{\mathrm{e}}=22.98\right]$, frequency $\left[F(3,138)=78.07, M S_{\mathrm{e}}=9.68\right]$, and cuing $\left[F(1,46)=18.31, M S_{\mathrm{e}}=3.46\right]$. No interactions approached significance.

A separate analysis on cued recall yielded significant effects of frequency $\left[F(3,69)=119.72, M S_{\mathrm{e}}=2.55\right]$ and cuing $\left[F(1,23)=7.73, M S_{\mathrm{e}}=3.30\right]$. The analysis of frequency estimates showed a similar pattern, finding significant effects of frequency $\left[F(3,69)=65.35, M S_{\mathrm{e}}=\right.$ $7.14]$ and cuing $\left[F(1,23)=10.65, M S_{\mathrm{e}}=3.62\right]$. The interaction between frequency and cuing was not significant in either analysis.

As in the earlier experiments, analyses were also done on two measures of accuracy for the frequency estimates. There were fewer categories given the correct estimate for cued (.31) than for noncued (.35) categories, but this difference fell just short of statistical significance $\left[F(1,23)=2.70, M S_{\mathrm{e}}=0.75, p<.10\right]$. There was a significant difference in unsigned deviations between cued (1.03) and noncued $(.92)$ categories $[F(1,23)=5.09$, $\left.M S_{\mathrm{e}}=4.45\right]$.

\section{GENERAL DISCUSSION}

The effects of generation, exemplar spacing, and extralist cuing on cued recall and categorical frequency estimates were studied in these experiments. These variables affected the magnitude and accuracy of frequency estimates, as well as the number of exemplars recalled. The results are consistent with an exemplar-retrieval account.

Alba et al . (1980) and Brooks (1985) argued that categorical frequency estimation is based upon a category frequency counter that is incremented automatically whenever an exemplar is encountered. The assumption of automatic incrementing is challenged by the results of Experiments 1 and 2, in which estimates were shown to be affected by variables influencing rehearsal and organizational processes. Even more fundamentally, the results 
of Experiment 3 present difficulties for any theory that would attribute estimates solely to the use of information stored at the superordinate level. Extralist cues lowered the frequency and magnitude of estimates, even though they could hardly have disrupted access to the superordinate level since category names were supplied at time of test. It is possible that a category counter could have mistakenly been incremented when extralist exemplars were accounted on the test. However, this should have led to an increase in frequency estimates for the cued categories, not the decrease that was found. These results suggest that the retrieval of exemplars plays an important role in the process of estimating categorical frequency.

Future research will be needed to determine how generally applicable these conclusions are. The strategy that subjects follow in categorical frequency estimation may depend upon factors such as the number of exemplars per list and the instructions given by the experimenter. Under the conditions used here, the exemplar-retrieval approach seems to be an accurate description of the processes underlying categorical frequency estimation.

These results converge with conclusions reached from experiments in which individual items are repeated exactly. A multiple-trace approach (Hintzman, 1976), in which each occurrence of a repeated item leaves an independent trace, has long been thought a viable account of repetition effects. Experiments involving item frequency estimation have offered support for the multipletrace approach (e.g., Begg, Maxwell, Mitterer, \& Harris, 1986; Greene, 1984, 1986, 1988; Hintzman, Grandy, \& Gold, 1981; Hintzman, Nozawa, \& Irmscher, 1982; Hintzman \& Stern, 1978). The experiments reported here suggest that a similar approach, one emphasizing the formation and retrieval of individual traces, best accounts for categorical frequency estimation.

\section{REFERENCES}

Alba, J. W., Chromiak, W., Hasher, L., \& Attig, M. S. (1980) Automatic encoding of category size information. Joumal of Experimental Psychology: Human Learning \& Memory, 6, 370-378.

BARsalou, L. W., \& Ross, B. H. (1986). The roles of automatic and strategic processing in sensitivity to superordinate and property frequency. Journal of Experimental Psychology: Learning, Memory, \& Cognition, 12, 116-134.

Battig, W., \& Montague, W. (1969). Category norms for verbal items in 56 categories: A replication and extension of the Connecticut category norms. Journal of Experimental Psychology Monographs, 80(3, Pt. 2).

BegG, I., MaXwell, D., Mitterer, J. O., Harris, G. (1986). Estimates of frequency: Attribute or attribution? Journal of Experimental Psychology: Learning, Memory, \& Cognition, 12, 496-508.

BEGG, I., \& SNIDER, A. (1987). The generation effect: Evidence for generalized inhibition. Journal of Experimental Psychology: Learning, Memory, \& Cognition, 13, 553-563.

BrooKs, J. E. (1985). Judgments of category frequency. American Journal of Psychology, 98, 363-372.

GREENE, R. L. (1984). Incidental learning of event frequency. Memory \& Cognition, 12, 90-95.
Greene, R. L. (1986). The effects of intentionality and strategy on memory for frequency. Journal of Experimental Psychology: Learning, Memory, \& Cognition, 12, 489-495.

GREENE, R. L. (1988). Generation effects in frequency judgment. Journal of Experimental Psychology: Learning, Memory, \& Cognition, 14, 298-304.

HANSON, C., \& HIRST, W. (1988). Frequency encoding of token and type information. Journal of Experimental Psychology: Learning, Memory, \& Cognition, 14, 289-297.

Hintzman, D. L. (1976). Repetition and memory. In G. H. Bower (Ed.), The psychology of learning and motivation (Vol. II, pp. 47. 91. New York: Academic Press.

Hintzman, D. L. (1986). "Schema abstraction" in a multiple-trace memory model. Psychological Review, 93, 411-428.

Hintzman, D. L., Grandy, C. A. \& Gold, E. (1981). Memory for frequency: A comparison of two multiple-trace theories. Journal of Experimental Psychology: Human Learning \& Memory, 7, 231-240.

Hintzman, D. L., Nozawa, G., \& Irmscher, M. (1982). Frequency as a nonpropositional attribute of memory. Journal of Verbal Learning \& Verbal Behavior, 21, 127-141.

Hintzman, D. L., \& STern, L. D. (1978). Contextual variability and memory for frequency. Journal of Experimental Psychology: Human Learning \& Memory, 4, 539-549.

HUNT, K. P., HodGe, M. H. (1971). Category-item frequency and category-name meaningfulness $\left(\mathrm{m}^{\prime}\right)$ : Taxonomic norms for 84 categories. Psychonomic Monograph Supplements, 4(6, Whole No. 54), 97-121.

McFarland, C. E., Jr., Frey, T. J., \& Rhodes, D. D. (1980). Retrieval of internally versus externally generated words in episodic memory. Journal of Verbal Learning \& Verbal Behavior, 19, 210-255.

Medin, D. L., \& Schaffer, M. M. (1978). Context theory of classification learning. Psychological Review, 76, 165-178.

Mueller, C. W., Watkins, M. J. (1977). Inhibition from part-set cueing: A cue-overload interpretation. Journal of Verbal Learning \& Verbal Behavior, 16, 699-709.

MURPhy, M. D., \& PufF, C. R. (1982). Free recall: Basic methodology and analysis. In C. R. Puff (Ed.), Handbook of research methods in human memory and cognition (pp. 99-128). New York: Academic Press.

Nairne, J. S., Pusen, C., Widner, R. L., JR. (1985). Representation in the mental lexicon: Implications for theories of the generation effect. Memory \& Cognition, 13, 183-191

NaIRNe, J. S., W WDNER, R. L., JR. (1987). Generation effects with nonwords: The role of test appropriateness. Journal of Experimental Psychology: Learning, Memory, \& Cognition, 13, 164-171.

Nickerson, R. S. (1984). Retrieval inhibition from part-set cuing: A persisting enigma in memory research. Memory \& Cognition, 12 , 531-552.

PufF, C. R. (1974). A consolidated theoretical view of stimulus-list organization effects in free recall. Psychological Reports, 34, 275-288.

Slamecka, N. J., \& Graf, P. (1978). The generation effect: Delineation of a phenomenon. Journal of Experimental Psychology: Human Learning \& Memory, 4, 592-604.

Slamecka, N. J., \& Katsaiti, L. T. (1987). The generation effect as an artifact of selective displaced rehearsal. Joumal of Memory \& Language, 26, 589-607.

TVersky, A., Kahneman, D. (1973). Availability: A heuristic for judging frequency and probability. Cognitive Psychology, 5, 207-232.

W ATKINS, M. J. (1975). Inhibition in recall with extralist "cues." Journal of Verbal Learning \& Verbal Behavior, 14, 294-303.

Williams, K. W., DuRso, F. T. (1986). Judging category frequency: Automaticity or availability? Journal of Experimental Psychology: Learning, Memory, \& Cognition, 12, 387-396.

(Manuscript received June 29, 1988; revision accepted for publication September 29, 1988.) 